\title{
Nature-Inspired Flow-Fields and Water Management for PEM Fuel Cells
}

\section{Jason In Sung Cho ${ }^{1,2}$, Toby Neville ${ }^{1,2}$, Panagiotis Trogadas ${ }^{1}$, Billy Wu ${ }^{3}$, Dan Brett ${ }^{1,2}$ and Marc-Olivier Coppens ${ }^{1}$}

${ }^{1}$ Department of Chemical Engineering, University College London, London WC1E 7JE, United Kingdom,m.coppens@ucl.ac.uk

${ }^{2}$ Electrochemical Innovation Lab, Department of Chemical Engineering, University College London, London WC1E 7JE, United Kingdom

${ }^{3}$ Department of Mechanical Engineering, Imperial College London, UK

Flow-field design is crucial to polymer electrolyte membrane fuel cell (PEMFC) performance, since non-uniform transport of species to and from the membrane electrode assembly (MEA) results in significant power losses ${ }^{[1]}$. The long channels of conventional serpentine flow-fields cause large pressure drops between inlets and outlets, thus large parasitic energy losses and low fuel cell performance ${ }^{[2]}$.

Here, a lung-inspired approach is used to design flow fields guided by the structure of a lung. The fractal geometry of the human lung has been shown to ensure uniform distribution of air from a single outlet (trachea) to multiple outlets (alveoli). Furthermore, the human lung transitions between two flow regimes: 14-16 upper generations of branches dominated by convection, and 7-9 lower generations of space-filling acini dominated by diffusion ${ }^{[3,4]}$. The upper generations of branches are designed to slow down the gas flow to a rate compatible with the rate in the diffusional regime (Pé $\sim 1)^{[5]}$, resulting in uniform distribution of entropy production in both regimes ${ }^{[3,4]}$.

By employing a three-dimensional (3D) fractal structure as flow field inlet channel, we aim to yield similar benefits from replicating these characteristics of the human lung. The fractal pattern consists of repeating " $\mathrm{H}$ " shapes where daughter " $\mathrm{H}$ 's" are located at the four tips of the parent "H". The fractal geometry obeys Murray's law, much like the human lung, hereby leading to minimal mechanical energy losses. Furthermore, the three-dimensional branching structure provide uniform local conditions on the surface of the catalyst layer as only the outlets of the fractal inlet channel are exposed to the MEA. 
Numerical simulations were conducted to determine the number of generations required to achieve uniform reactant distribution and minimal entropy production. The results reveal that the ideal number of generations for minimum entropy production lies between $N=5$ and $7^{[6]}$. Guided by the simulation results, three flow-fields with $N=3,4$ and $5\left(10 \mathrm{~cm}^{2}\right.$ surface area) were 3D printed via direct metal laser sintering (DMLS), and experimentally validated against conventional serpentine flow fields. The fractal flow fields with $N=4$ and 5 generations showed $\sim 20 \%$ and $\sim 30 \%$ increase in performance and maximum power density over serpentine flow fields above $0.8 \mathrm{~A} \mathrm{~cm}^{-2}$ at $50 \% \mathrm{RH}^{[6]}$. At fully humidified conditions, though, the performance of fractal $N=5$ flow-field significantly deteriorates. The susceptibility towards flooding of the high-generation fractal flow-fields is due to the lack of convective gas flow requisite for effective liquid water removal, resulting in significant water accumulation in the interdigitated outlet channels. This was recently confirmed using neutron radiography in an in situ water visualisation study.

Another defining characteristic of the fractal approach is scalability, which is an important feature in nature. Fractal flow-fields can bridge multiple length scales by adding further generations, while preserving the building units and microscopic function of the system. Larger, 3D printed fractal flow-fields (25 $\mathrm{cm}^{2}$ surface area) with $N=4$ are compared to conventional serpentine flow-field based PEMFCs. Performance results show that fractal and serpentine flow-field based PEMFCs have similar polarization curves, which is attributed to the significantly higher pressure drop $(\sim 25 \mathrm{kPa})$ of large serpentine flow fields compared to fractal flow-fields. However, such excessive pressure drop renders the use of a large scale serpentine flow field prohibitive, thus favouring the fractal flow-field.

Lastly, a novel water management strategy that draws inspiration from the passive directional liquid water transport across the skin of the thorny devil is presented. The thorny devil is an Australian lizard with ridged scales, which collects water from any part of its body by simply touching water. The water is transported to the mouth across the skin via capillary action. The phenomenon is based on geometric principles, namely on a periodic pattern of interconnected capillary channels ${ }^{[7]}$. Using a one-step processing technique, we reproduced the passive water transport phenomenon through fabrication of interconnected capillary channels on the surface of graphite flow field. Preliminary results indicate more than a twofold improvement in fuel cell power density with the integration of capillary channels onto the surface of a parallel flow field, due to superior liquid water separation and removal. Implementation of this water 
management strategy could circumvent remaining problems of high-generation fractal flow fields.

\section{References}

[1] $\mathrm{Wu}, \mathrm{H} .-\mathrm{W} .$, A review of recent development: Transport and performance modeling of PEM fuel cells. Applied Energy, 2016. 165: p. 81-106.

[2] Hsieh, S.-S., B.-S. Her, and Y.-J. Huang, Effect of pressure drop in different flow fields on water accumulation and current distribution for a micro PEM fuel cell. Energy Conversion and Management, 2011. 52(2): p. 975-982.

[3] Kjelstrup, S., Coppens, M.-O., Pharoah, J., and Pfeifer, P., Nature-inspired energy-and material-efficient design of a polymer electrolyte membrane fuel cell. Energy \& Fuels, 2010. 24(9): p. 5097-5108.

[4] Trogadas, P., Ramani, V., Strasser, P., Fuller, T.F., and Coppens, M.-O., Hierarchically Structured Nanomaterials for Electrochemical Energy Conversion. Angewandte Chemie International Edition, 2016. 55(1): p. 122-148.

[5] Coppens, M.-O., A nature-inspired approach to reactor and catalysis engineering. Current Opinion in Chemical Engineering, 2012. 1(3): p. 281-289.

[6] Trogadas, P., Cho, J.I.S., Neville, T.P., Marquis, J., Wu, B., Brett, D.J.L., and Coppens, M.-O., A lung-inspired approach to scalable and robust fuel cell design. Energy \& Environmental Science, 2018. 11(1): p. 136-143.

[7] Comanns, P., Effertz, C., Hischen, F., Staudt, K., Böhme, W., Baumgartner, Directional, passive liquid transport: the Texas horned lizard as a model for a biomimetic 'liquid diode'. Journal of The Royal Society Interface, 2015. 12(109). 